Brit. J. industr. Med., 1960, 17, 46.

\title{
THE DEVELOPMENT OF ROTARY NYSTAGMUS IN THE CAT AS A FUNCTION OF AGE
}

\author{
BY \\ N. E. LOVELESS \\ From the Nuffield Department of Industrial Health, University of Durham \\ (King's College, Newcastle upon Tyne)
}

(RECEIVED FOR PUBLICATION FEBRUARY 3, 1959)

\begin{abstract}
Although it is known that the onset of miners' nystagmus is related to the age of the worker, the nature of the relationship is difficult to determine from clinical data. An attempt has been made to clarify this relationship by the use of animal subjects. Thirty-five cats of ages ranging from 1 to 41 weeks were kept in darkness and examined regularly for oscillation of the eyeballs. The rate at which the amplitude of oscillations increased appeared to be a logarithmic function of the age at which the animal was placed in darkness. This result is discussed in relation to the mechanisms involved in the disease. A second important finding is that every animal tested developed detectable oscillations. It is suggested that the same would be true of man, given sufficient exposure to conditions of low illumination. This raises more acutely the question why incapacity resulting from the disease is found in only a minority of miners, and is not clearly related to the severity of the visual disorder.
\end{abstract}

The onset of miners' nystagmus appears to be related in some way to the age of the worker. It is commonly held to be a disease of middle age, occurring most typically after some 20 to 30 years of underground work. This statement is presumably based upon the first report of the Miners' Nystagmus Committee (1922), which includes a graph showing the ages of 2,000 certified cases at the time of onset of the disease. While this distribution ranges over the entire span of working life, it approximates closely to a normal distribution with a median age of about 40 years and a quartile deviation of about eight years. Thus, from the clinician's point of view, it is true to say that certifiable cases will most typically be men in early middle life.

This statement can, however, be misleading in that it takes no account of the fact, shown in the same graph, that the number of men employed underground decreases progressively with age. If the data given are assumed to be representative, and are replotted to show incidence relative to the proportion of men employed underground in each age group, the resulting curve (Fig. 1) shows no distinct peak in the middle years, but is rather a monotonic and positively accelerated function of age. It might be regarded as the early section of a normal distribution, but in this case the peak presumably falls beyond the span of working life. Thus, from the point of

view of the worker rather than the clinician, the risk that he will be affected by the disease appears

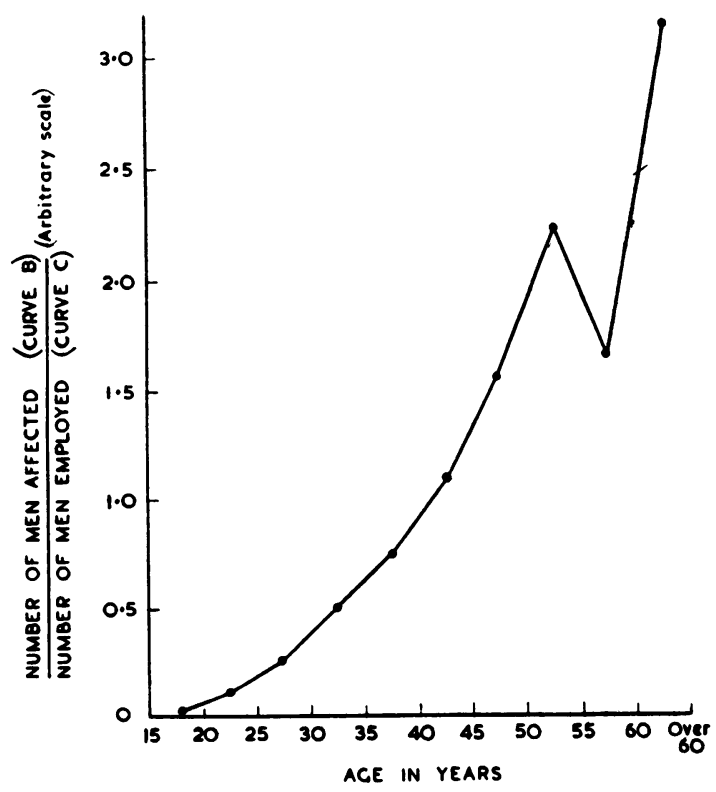

Fig. 1.-Data from Graph V of first report of Miners' Nystagmus Research Committee (1922) re-plotted to show incidence relative to numbers in each age group employed underground. 
to increase continuously as long as he remains exposed to the conditions of work which produce it. It should also be noted that we cannot confidently set any limit to the proportion of men who might theoretically be affected if the working span were sufficiently prolonged.

A number of factors make it difficult to determine the relationship between age and the development of nystagmus from clinical data. There are obvious statistical deficiencies in the available data: the progressive retirement with age from work at the coalface may well be selective; the conditions of illumination in which a man has worked are not exactly known, and since the majority of miners begin work in adolescence, the effect of age cannot be separated from that of time spent underground.

The present study is an attempt to control these factors by the use of animal subjects, and so to determine the effect of age on the development of nystagmic oscillations.

\section{Procedure}

The subjects of the experiment were 37 cats. At predetermined ages between 1 and 41 weeks, they were placed in individual cages in an experimental room, where they were kept in total darkness except for a brief daily period during which they were cleaned and fed. An adequate diet and a reasonable standard of heating and ventilation were provided, but despite these precautions, two cats died of a virus infection during the course of the experiment. Complete records are therefore available only for the remaining 35 animals, which were kept in good health, apart from some muscular atrophy due presumably to lack of exercise.

The animals were inspected weekly to determine whether oscillations could be detected, first with the naked eye, then with an ophthalmoscope, and these two observations were separately recorded. The difference between the two criteria is essentially one of amplitude, since the ophthalmoscope made possible the detection of finer oscillations. It was, however, more difficult to distinguish these from the effects of head tremor and voluntary eye movement, and the experimenter felt more confidence in judgments made with the naked eye. The ophthalmoscope was also difficult to use with very young kittens because of the opacity of the optic media, the small aperture of the pupil, and the subjects' constantly changing direction of gaze.

Observations were discontinued on animals which did not produce oscillations visible with the naked eye after two to three years in darkness. The remaining animals were kept in daylight after various periods spent in darkness, and observations were made on the course of their recovery.

\section{Analysis of Data}

An estimate was made of the period spent in darkness before each of the two criteria was reached. The younger cats normally progressed fairly steadily from a state in which no oscillations could be detected to one in which oscillations could be detected only with the ophthalmoscope, and finally to one in which they were visible to the naked eye. The date at which a transition took place can therefore be stated to the nearest week. As in man, however, the amplitude of oscillation shows some fluctuation with such factors as the animal's posture and direction of gaze, and probably with its emotional state. A small number of records, mostly of older cats, do not therefore show a steady progression from week to week. In these cases a time is stated after which the given criterion is recorded with a fair degree of consistency.

The element of uncertainty involved may be illustrated by the record of cat No. 42 (Table 1). This animal was placed in darkness at the age of 20 weeks. The record for the next 30 weeks reads as follows, where $\mathbf{S}$ denotes steadiness of the eye, $\mathrm{O}$ an oscillation detectable only with the ophthalmoscope, and $\mathrm{N}$ an oscillation detectable with the naked eye:-S S S S S S S S O O S O O O O O O N O O O O O N N N N N N. The onset of oscillations detectable with the ophthalmoscope was taken as from the ninth week, despite a later negative record, and the onset of oscillations detectable with the naked eye was taken as from the twenty-fourth week, despite an earlier positive record.

Where the records show more than a transitory irregularity, this has been indicated in the results.

\section{Results}

The estimates obtained by the above procedure are presented in Table 1. It will be seen that oscillations were detected with the ophthalmoscope in all the animals, but appeared to develop more quickly in the younger cats. The majority of animals up to 15 weeks old developed oscillations visible with the naked eye, but four cats, aged 6 to 8 weeks, failed to do so, and for three of these the ophthalmoscopic records are irregular. Of four animals between 20 and 25 weeks old, three showed only a transitory episode of oscillations visible with the naked eye. No animals over 25 weeks old reached this criterion after two to three years in darkness. In the majority of the older cats, the ophthalmoscopic records show some irregularity; in a number of cases, the oscillations become sporadic after a time, and occasionally seem to have disappeared altogether.

In Fig. 2, the time taken to develop oscillations visible with the ophthalmoscope is plotted as a function of the age at which the animal was placed in darkness, the ordinate being shown on a logarithmic scale. Although the data show a considerable scatter, they yield a rank-order correlation 
TABLE 1

TIME TO DEVELOP NYSTAGMUS AS A FUNCTION OF AGE OF CATS PUT IN DARKNESS

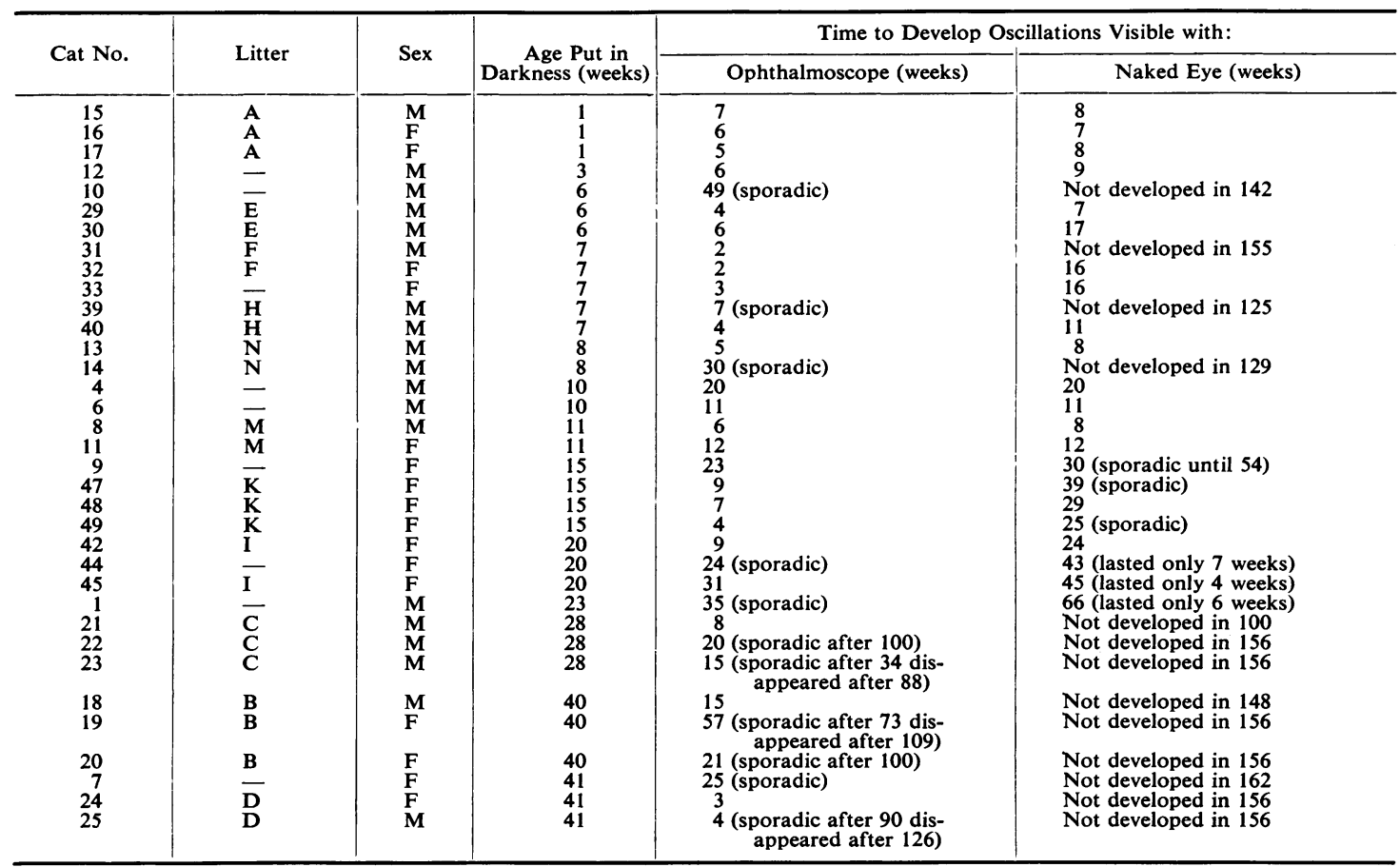

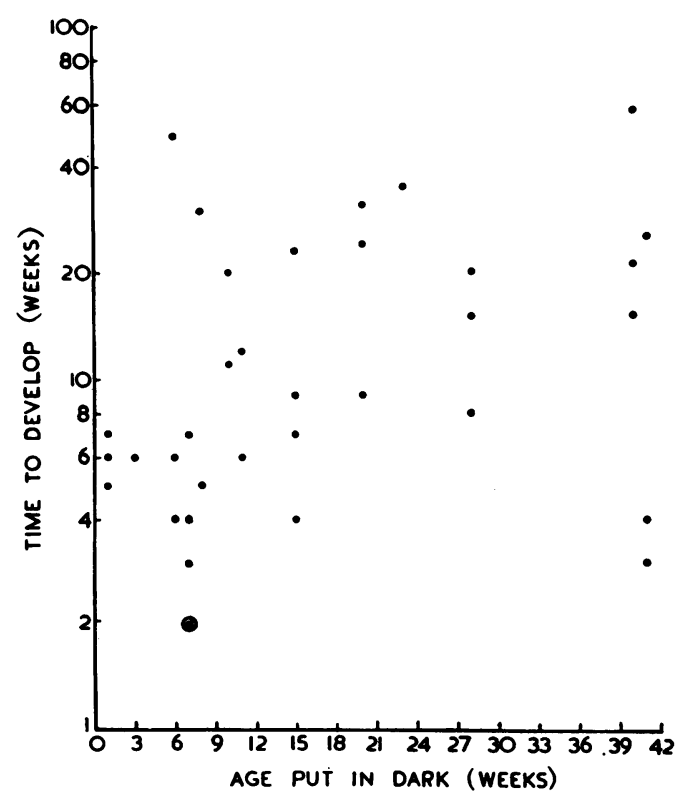

FIG. 2.-Time to develop oscillations detectable with the ophthalmoscope, as a function of age of cats put in darkness.

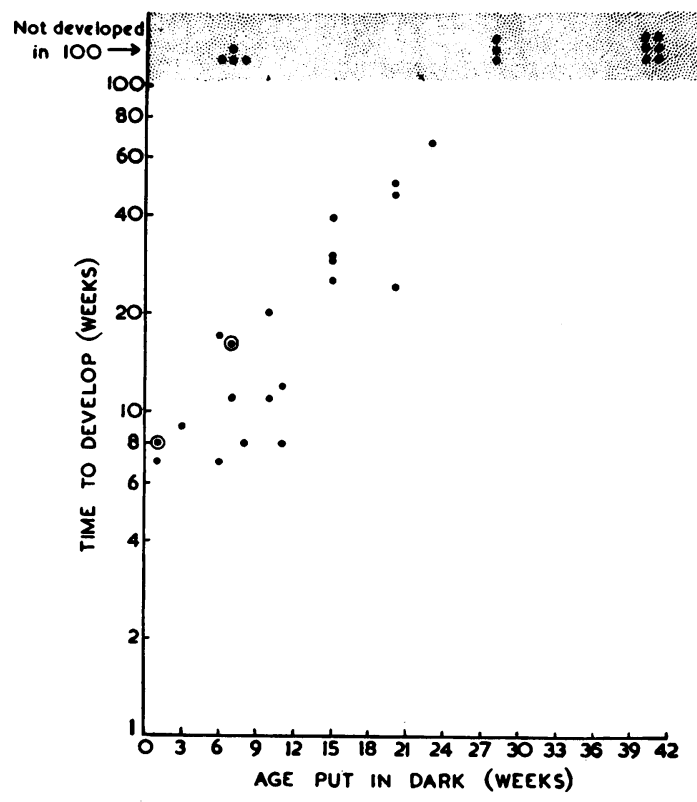

FIG. 3.-Time to develop oscillations detectable with the naked eye, as a function of age of cats put in darkness. 
coefficient of $+0 \cdot 39$, which is significant at the $2 \%$ level of confidence.

Fig. 3 shows the same relationship for the nakedeye criterion; the cats which developed no oscillations are shown at the top of the graph. If these cases are treated as an upper terminal category, the rankorder correlation coefficient is +0.69 , which is significant beyond the $0.001 \%$ level of confidence. The trend shown on the graph is reasonably linear, suggesting that the time taken to develop oscillations of a given amplitude increases logarithmically with age.

Details of recovery rates are not presented, since the number of cases is too small to establish any definite relationship when time spent in darkness must be considered as an additional variable. It may, however, be noted that while the great majority of cats recover completely within a year, the three animals which spent 25 weeks in darkness from the age of 1 week still show gross oscillations after more than five years in daylight.

\section{Discussion}

Nystagmic oscillations appear in general to increase in amplitude at a rate dependent upon the age of the animal when placed in darkness. The four cats aged 6 to 8 weeks, which did not develop oscillations large enough to be detected with the naked eye, seem sufficiently divergent from this general trend to constitute a separate group. We can at present offer no explanation of this anomaly. It is perhaps worth noting that all four animals were male, but scrutiny of the remaining cases does not suggest that sex has any general influence on the development of the condition. Each of the four animals came from a different litter.

The fact that the relationship appears to be nonlinear is of some interest. It suggests, for example, that a change in school leaving age might have a disproportionate effect upon the incidence of nystagmus, and that a measure of prevention might be attained by allocating the younger miners to the best available conditions of illumination. The plausibility of these suggestions rests, however, upon the extent to which visual development in the cat parallels that in man, and upon consideration of the mechanisms involved in the disorder.

Comparative studies of nystagmus have thrown some light on these mechanisms. Ohm (1916) produced nystagmus in dogs and cats kept in darkness, Riesen $(1947,1950)$ produced it in chimpanzees, and Browne (1951) was able to produce it in cats, but not in rats or rabbits. It is apparently not present in pit ponies. Susceptibility to nystagmus therefore appears to be related to the extent of the binocular field and of decussation at the optic chiasma, the ability to perform voluntary conjugate eye movements, and the possession of a diurnal eye with a well-marked area centralis. The latter feature may well be the critical factor, since the horse has a diurnal eye whose area of highest acuity forms a horizontal band rather than a central zone (Walls, 1942). In this connexion it is of interest to consider phylogenetic differences in optokinetic reactions. Eye movements in pursuit of moving striations can be elicited in vertebrates as low as the lizard, and appear to be subvortically determined. The pursuit of single objects against a dark background, however, appears to involve a different mechanism. Optokinetic reactions of this kind can be produced in dogs, cats, and monkeys, but not in rodents, and are abolished by destruction of the striate cortex. Since this operation also increases the extent of the striations of a patterned field required to elicit visual pursuit, it is plausible to suppose that it eliminates the central mechanisms of the area centralis. The absence of this type of optic nystagmus in rodents is then accounted for by their complete lack of an area of acute vision (Smith and Bojar, 1938). These facts accord with the hypothesis that miners' nystagmus involves disturbance of the normal process of fixation, as a result of life in conditions of illumination in which the macula cannot function (Davson, 1949; Ferguson, 1952). The failures of binocular vision seen in miners' nystagmus appear to be the result of oscillations rather than their cause (Campbell, Harrison, and Vertigen, 1951).

On this view, it would be expected that the effect of exposure to darkness would vary with the extent to which normal macular fixation has become established by maturation and learning. In the cat, an optokinetic response to moving striations first appears about the fourteenth day, and the width of striations necessary to produce it then decreases until about the twenty-sixth day. These developments precede the formation of precise localizing responses of the forelimbs and other behaviour requiring fine visual adjustment (Smith and Bojar, 1938). There is unfortunately no evidence on the ontogeny of optokinetic responses to single moving objects, but it is probable that movements of this type resemble binocular convergence and object recognition in requiring considerable practice for their perfection (Riesen, 1950). Those animals which have received most practice might then take longer to lose the habit when placed in darkness, and might more readily revert to it when returned to daylight. It is also likely, however, that maturational factors are of importance in the perfection of these responses. There is evidence that exposure to radiation in the first few weeks of life is necessary for the 
normal development of the retina and its associated structures. It has also been shown that chicks reared in darkness and artificially fed never develop the normal pecking response (Beach and Jaynes, 1954). Thus prevention of practice at the appropriate stage of growth may interfere with the development of behaviour which appears to be instinctive or reflex in nature. It seems possible that the three cats placed in darkness at the age of 1 week similarly failed to develop normal macular fixation.

A comparable process of development takes place in man. The human infant shows some evidence of monocular fixation from birth, but sustained voluntary fixation appears to require the development of an inhibitory mechanism which does not become efficient until about the age of 4 weeks. Binocular convergence is not clearly present until about the eighth week, and it is likely that the development of oculomotor skill continues for a much longer period (Gesell, Ilg, and Bullis, 1949). It may be doubted whether any appreciable change in performance is still occurring at adolescence, but the long reinforcement which the habit has then received makes it understandable that miners develop oscillations of appreciable magnitude only after considerable exposure to conditions of low illumination.

The present experiment appears to be the first comparative study which has used an appreciable number of subjects. It seems important, therefore, to stress that every animal produced detectable oscillations, at least for a period. This strongly supports the hypothesis that defective illumination is the primary cause of the visual disorder, and makes it reasonable to suppose that every miner working in sufficiently low illumination would develop oscillations sooner or later. This conclusion seems to be in accordance with the clinical evidence, when due allowance is made for variation in lighting standards. The Miners' Nystagmus Committee reported in 1923 that the prevalence of oscillations in miners working in safety-lamp pits might then be as high as $34 \%$ of the men employed. Caiger (1941) was of the opinion that an expert observer could detect oscillations in most miners over 50 years of age.

The probability that susceptibility to oscillations is universal raises more acutely the already muchdiscussed problem of the extent to which the visual disorder is responsible for incapacity to work. Since normal "physiological" nystagmus does not produce instability of the visual field, and is in fact essential for seeing, it might be supposed that oscillations must build up to some critical amplitude before they become disturbing; individual differences in the rate of development might then explain why most men do not reach this stage within their working lives. Against this is the fact that men with marked oscillations may be free of symptoms. Presumably such individuals are able to adapt their visual mechanisms so as to tolerate the disorder; that such adaptation is possible is clear from the stability of the visual field in congenital nystagmus. Again, men who are aware of their oscillations often develop some skill in controlling them (Campbell et al., 1951). Disablement might then be regarded as due to psychological incapacity to adapt, in one way or another, to the visual disorder. This hypothesis faces the difficulty that many certified nystagmics do not exhibit gross oscillations, but it seems possible that a considerable proportion of these suffer from defects of binocular vision which are not associated with oscillations, though they give rise to similar symptoms (Campbell et al., 1951). Adaptability is likely to be itself a function of age (Welford, 1958), as well as of personality.

Most investigators agree that there is a psychological element in the disease as certified, and psychiatric experts have usually held that this factor is predominant (Culpin, 1925; Halliday, 1943; Stern, 1948). More recently, objective psychological tests have shown that men with nystagmus are less emotionally stable than men without the disease (Lion, 1958a). This is not to deny that other physical factors may affect the development of the disease. There is evidence that the symptoms are rendered more likely by work in a stooping posture (Browne and Beck, 1954). Defective dark adaptation is also associated with the disorder, though its role is unclear (Ferguson, 1952). It should, however, be noted that this condition is itself associated with emotional instability (Eysenck, 1947).

The precise role of the psychological element has been the subject of some controversy. Thus, it has been suggested (Miners' Nystagmus Committee, 1923) that the terms of certification allow the inclusion of a number of neurotic individuals without gross visual symptoms; but the psychological tests show no difference in emotional stability between certified nystagmics with and without oscillations (Lion, 1958b).

A further possibility is that the psychiatric features of the disorder are a not unjustified reaction to the disturbing visual symptoms and the threat of unemployment and financial hardship. Browne, Beck, Saint, and McCallum (1950) describe the nystagmic patient as a conscientious, quiet, family man. An individual who takes his responsibilities seriously might be regarded as more likely to react with anxiety and depression to the onset of visual symptoms.

It is not certain that the relationship of visual and emotional symptoms operates only in one direction. 
It has been suggested that patients are more likely to exhibit oscillations when in a state of excitement (Shufflebotham, 1914). We believe this to be true of our experimental animals also, as exaggerated oscillations may sometimes be seen in animals startled by an extraneous noise in the course of examination. It would be of value to confirm these observations by experiment, for example, by studying the effects of an injection of adrenalin.

If emotional disturbance does increase the amplitude of oscillations, these two factors might well interact cumulatively until a breakdown is precipitated, once the visual disorder has progressed sufficiently to come to the patient's notice.

This stage may also be reached earlier in those subjects most predisposed to anxiety. Miners in general appear to show more evidence of emotional stress than the general population (Heron and Braithwaite, 1953; Lion, 1959), which is hardly surprising in view of the hazards of the occupation. Poor lighting may add to this stress, and work in conditions of reduced sensory input may in itself induce psychological disturbance (Bexton, Heron, and Scott, 1954). Those most affected by this stress may therefore develop a given amplitude of oscillation more rapidly, and the rate of development may well affect the possibility of adaptation to the disorder.

The difficulty of determining the influence of predisposition in a human population is evident, particularly when the disorder develops as slowly as in nystagmus. The possibility of investigating this aspect of the problem with animal subjects is there- fore worth considering, as some progress has been made in developing measures of emotionality in animals (Broadhurst, 1957, 1958).

The author has had the advantage of discussing this paper with Professor R. C. Browne, Dr. R. I. McCallum, Mr. D. J. Newell, and Mr. D. H. Holding. The successful completion of the work owes much to the standard of animal husbandry maintained by Mr. G. E. South and his assistants.

\section{REFERENCES}

Beach, F. A., and Jaynes, J. (1954). Psychol. Bull., 51, 239. Bexton, W. H., Heron, W., and Scott, T. H. (1954). Canad. J. Psychol., 8, 70.

Broadhurst, P. L. (1957). Brit. J. Psychol., 48, 1.

(1958). Ibid., 49, 12

Browne, R. C. (1951), Lancet, 1, 721.

- and Beck, I. F. (1954). Brit. med. J., 1, 1176

-, Saint, E. G., and McCallum, R. I. (1950). Brit. J. Ophthal., 34, 601.

Caiger, H. (1941). Med. Press, 206, 152.

Campbell, Harrison, R., and Vertigen, J. (1951). Brit. J. Ophthal., 35, 484.

Culpin, M. (1925). Ibid., 9, 564

Davson, H. (1949). The Physiology of the Eye. Churchill, London.

Eysenck, H. J. (1947). Dimensions of Personality. Kegan Paul, Trench, Trubner and Co., London.

Ferguson, W. J. W (1952) Trans. III. Engng Soc. 17, 49.

Gesell, A., Ilg, F. L., and Bullis, G. E. (1949). Vision: its Development in Infant and Child. Hoeber, New York.

Halliday, J. L. (1943). Psychosom. Med., $5,71$.

Heron, A., and Braithwaite, D. (1953). Brit. J. industr. Med., 10 27.

Lion, J. S. (1958a). Ibid., 15, 204. (1958b). Brit. med.J., 2,

(1959). To be published. (1922). Spec, Rep. Ser, med. Res. Coun. (Lond.), No. 65 .

Ohm, J. (1916). Z̈. Augenheilk., 36, 198.

Riesen. A. H. (1947). Science, 106, 107.

Riesen, A. H. (1947). Science, 106, 107.

Shufflebotham, F. (1914). Brit. med. J., 1, 648 .

Smith, K. U., and Bojar, S. (1938). Psychol. Bull., 35, 193.

Stern, E. S. (1948). Brit. J. Ophthal., 32, 209.

Walls, G. L. (1942). The Vertebrate Eye. Cranbrook Inst. Sci. (Institute of Science), Bloomfield Hills, Michigan.

Welford, A. T. (1958). Ageing and Human Skill. Oxford University Press, London. 